${ }^{1}$ Alikulov Z.A., ${ }^{2 *}$ Shalakhmetova G.A., ${ }^{2}$ Aytasheva Z.G., ${ }^{2}$ Zhussupova A.I., ${ }^{2}$ Balmukanov A.U.

${ }^{1}$ L.N.Gumilyev Eurasian National University, Astana, Kazakhstan, ${ }^{2}$ al-Farabi Kazakh National University, Almaty, Kazakhstan *e-mail: Galiya.Shalakmetova@kaznu.kz

\title{
Nitrate reductase in detached embryos may serve as a marker of the preharvest tolerance of wheat seeds
}

\begin{abstract}
We have observed an easily determinable parameter indicative of genetic pre-harvest sprouting (PHS) tolerance - the presence of an endosperm factor, presumably ABA, capable of inhibiting nitrate reductase (NR) induction in the embryo in the presence of $\mathrm{NO}_{3}$. This finding has importance not only for the early rapid screening of PHS tolerant of cereal cultivars but may also be an important tool to determine the mechanism of NR inhibition either by genetic repression or by post-translational down regulation of NR activity. In this work we sought a simple relationship between ABA content and NR activity level that we assumed to be closely related to PHS susceptibility. Studied the relationship between ABA content, dry weight and ability to germinate in seeds of examined wheat varieties resistant to preharvest sprouting Lutescens 70 , and unstable - Novosibirskaya 67 . The data obtained are shown that the level of ABA reaching its maximum to 40-45 days. The maximum content of ABA for both varieties accounted for 40 days after flowering, when the grains reach maximum wet weight in the future, as the ripening hormone levels quickly decreased with the decrease in fresh weight of grain. The level of ABA in embryos unstable to pre-harvest sprouting wheat Novosibirskaya 67 ranged from 20 to $40 \%$ below the level of the hormone in the grains resistant Lutescens 70 wheat. The results showed a significant increase in the ability to germinate, since it is a phase 40-45 DAP. Reduction of endogenous ABA content in grains at the final stage of maturation, with a relatively high percentage of germination, can be explained by the need and ensuring to the start of the germination process of the seed.
\end{abstract}

Key words: Pre-harvest sprouting (PHS), nitrate reductase (NR), abscisic acid (ABA), wheat seeds, PHS tolerant cultivar of wheat.

\section{Introduction}

Pre-harvest germination (PHS) is the widespread phenomenon resulting in inferiority of grain, to decrease in its consumer qualities and respectively the prices. The phenomenon of pre-harvest germination depends on many reasons and, first of all, from genetic signs of wheat, from a phytohormone of abscisic acid (ABA) and the gibberellic acid (GA), germinations regulating process dormancy of seeds, and also from climatic factors such as humidity, low temperatures, during the period maturing grains, and from existence in the soil of enough of Mo for growth and viability of plants. Precocious germination of wheat grain is a serious problem in wheat production. The phenomenon of germination of physiologically mature cereal grains in the ear or panicle, usually under wet conditions shortly before harvest, is termed as pre-harvest sprouting (PHS) or vivipary. PHS occurs in many cereal crops such as wheat, barley, maize, and rice in most region of the world. PHS not only causes reduction of grain yield, but also affects the quality of grains, resulting into significant economic losses. Therefore the physiological, genetic, and environmental basis of PHS susceptibility in wheat have been a subject of intensive research during the past three decades [1].

$\mathrm{ABA}$, a plant growth regulator, plays a pivotal role in the establishment of primary dormancy. ABA in mature grain has been shown to prolong dormancy in wheat by blocking germination of both the whole caryopses and the embryos. ABA is not only confined to mature grain, and it has been shown by Derera [2], that ABA levels in rise grain increase during their development. Therefore, $\mathrm{ABA}$ acts prior to maturity to inhibit germination. The transient rise in $\mathrm{ABA}$ content at approximately mid-development coincides with the establishment of dormancy. However, it also coincides with several other major events in seed development, such as dehydration, solidification of endosperm, testa formation and seed abscission $[3,4]$. A major step forward in seed dormancy research was 
made with the introduction of ABA-deficient mutants of Arabidopsis, maize, pea and tomato. Many of these mutants have in common lack of dormancy Gibberellic acid (GA) induces seed germination. GA increases activity of enzymes such as $\alpha$-amylase, protease, hemicellulase and more. ABA blocks GA induced gene transcription and enzyme production, while at the same time it stimulates the production of specific proteins, such as LEAs and the accumulation of anthocyanins and transcripts encoding storage proteins [4].

In wheat, barley, and sorghum cultivars that are susceptible to pre-harvest sprouting, there is a relative sensitivity of the embryo to ABA as compared to resistant cultivars, generally in the absence of any substantial differences in $\mathrm{ABA}$ content, or a higher $\mathrm{ABA}$ content in the susceptible cultivars $[5,6]$. Interestingly, the expression pattern of the sorghum VP1 gene is different in two cultivars genotypes exhibiting differential resistance to preharvest sprouting at physiological maturity. More specifically, in the embryos of the susceptible cultivar, transcripts encoding VP1 peak at a relatively early stage of grain development (20 days after pollination), whereas the peak in the resistant cultivar occurs at much later developmental stages, when seed maturation is almost complete [5]. Tanner showed that PHS in corn was linked with deficiency of molybdenum [6]. Premature germination in maize was eliminated by Mo applications to the soil or the leaves. Foliar application of Mo to wheat growing in sand led to increased seed dormancy $[7,8]$ and the concomitant increase of $\mathrm{ABA}$ content in newly produced seeds.

In higher plants, $\mathrm{ABA}$ is derived from an epoxycarotenoid precursor that is oxidatively cleaved to produce xanthoxin $[9,10]$. Following the cleavage, xanthoxin is converted to ABA by a series of ring modifications to yield abscisic aldehyde, which is oxidized to ABA by AO (EC 1.2.3.14), a molybdenumcontaining enzyme $[11,12]$. In addition to $\mathrm{AO}$, plant MoCo-containing enzymes include nitrate reductase (NR; EC 1.7.1.1) and XDH (EC 1.17.1.4) [13]. XDH and $\mathrm{AO}$ have been characterized from various organisms and shown to be homodimers of $150-\mathrm{kDa}$ subunits that have a high similarity in their amino acid sequence and motifs, among which are the binding sites for two iron-sulfur centers and a MoCo binding region $[14,15,16]$. Whereas, NR requires a dioxomolybdenum, center XDH and AO contain monooxoMoCo in which the second oxygen was replaced by a sulfur ligand.

It has been shown that mutations of genes in synthesis of the carotenoid precursors of ABA resulted in the pre-harvest sprouting, which is consequence of
ABA deficiency. The phenomenon is due to the lack of ABA in the young seeds and/or their insensitivity to the dormancy-inducing hormone [17].

\section{Materials and methods}

Experiments to determine the activities of Moenzymes as early markers of PHS were carried out in PHS tolerant (Latescence 70) and sensitive (Novosibirskaya 67) wheat varieties during seed germination. Wheat seeds of these varieties were obtained from the N. Baraev Cereal Research Institute at Shortandy (Kazakhstan). Preparation of seed tissue extracts and the activities of molybdoenzymes, aldehyde oxidase $(\mathrm{AO})$, xanthine dehydrogenase $(\mathrm{XDH})$ and nitrate reductase (NR) in the aleurone layers, endosperms and embryos were determined according to $[18,19]$.

NR activity was determined in vitro according to Savidov et al. [18] . The reaction medium contained $50 \mathrm{mM} \mathrm{Na}$-phosphate buffer ( $\mathrm{pH}=7.7), 5 \mathrm{mM} \mathrm{KNO}_{3}$ and $10 \mu \mathrm{M}$ FAD. The reaction was started by the addition of the enzyme (i.e., seed part extract). After 10 min of incubation at $28^{\circ} \mathrm{C}$, the reaction was terminated by adding $50 \mu \mathrm{l}$ of a mixture of $0.3 \mathrm{mM}$ phenazinemethosulfate (PMS) and $1 \mathrm{M}$ Zn acetate (1:1) to remove residual NADPH from the assay medium. This step was followed by the addition of $0.1 \mathrm{ml}$ of a mixture of $0.02 \%$ (NED) and $1 \%$ (SA) (1:1) for nitrite determination. Reaction mixtures were centrifuged at $10000 \mathrm{rpm}$ for $5 \mathrm{~min}$, and their absorbance was read at $540 \mathrm{~nm}$ after $20 \mathrm{~min}$.

AO activity was detected in $7.5 \%$ polyacrylamide gels after protein fractionation by native gel electrophoresis using indole-3-aldehyde as substrates for AO according to [19]. The samples, which contained approximately $20-25 \mu \mathrm{g}$ of soluble protein, were loaded onto wells and carry out electrophoresis at $4^{\circ} \mathrm{C}$ for $1.5-2$ hours. Then, the gels were equilibrated and washed in $100 \mathrm{mM}$ sodium phosphate buffer $(\mathrm{pH}=7.5)$ by gentle shaking for $10 \mathrm{~min}$, which was followed by incubation in a reaction mixture containing $0.1 \mathrm{mM}$ PMS, $1 \mathrm{mM}$ 3(4,5-dimethylthiazolyl-2)2,5-diphenyltetrazolium-bromide (MTT) and $1 \mathrm{mM}$ specific XDH/AO substrate. The activities of $\mathrm{XDH}$ and $\mathrm{AO}$ were estimated based on the amount of MTT reduction that resulted in the development of specific formazan bands. The intensity of formazan bands was directly proportional to enzyme activity in the presence of excess substrate and tetrazolium salt. Quantitative analyses were made by scanning the formazan bands in the gel with a computing laser densitometer (Molecular Dynamics) using the software provided (Image Quant version 3.19.4). 


\section{Results and their discussion}

Mo-enzyme activities in the parts of dry wheat seeds. The activities of NR and AO were determined in different parts of dry dormant seeds - the embryo, endosperm and aleurone layer. The embryo and aleurone layer extracts showed AO activity while these activities were not detected in the endosperm

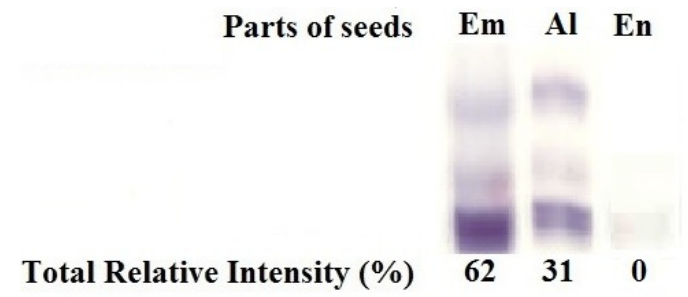

Figure 1 - The activity of $\mathrm{AO}$ in different parts of dormant seeds from the wheat cultivar Lutescens 70 (Em - embryo, $\mathrm{Al}$ - aleurone layer, En - endosperm)
Dry seeds were incubated in distilled for $24 \mathrm{~h}$, after which only the embryo showed no NR activity. NR activity developed in seed embryos only after incubation in the presence of $\mathrm{KNO}_{3}$ and its levels in embryos of both wheat varieties were nearly the same (not shown). Early results showed that NR-antibodies did not cross-react with protein of extracts of wheat seed embryos, endosperm and aleurone layer [20].

Mo-enzymes in developing and mature seeds. The activities of NR, XDH and AO in developing seeds were determined at ten days intervals starting from 10th day after pollination (DPA) (Table 2). Water content of the seed attained a peak at 30 DPA and then started declining to its lowest level a maturity. A linear increase in fresh as well as dry matter of the seed was observed between 10 and 30 DPA (not shown). XDH activity in the embryo and aleurone layer in maturing seeds of both varieties remained at a steady level. A slow increasing activity of AO reached a maximum at 40 DPA (Table 2).

Table 1 - Activities of AO, XDH and NR in different seed parts of PHS-tolerant cultivar Lutescence 70

\begin{tabular}{|c|c|c|c|}
\hline Seed parts & $\mathrm{AO}^{1}$ & $\mathrm{XDH}^{2}$ & $\mathrm{NR}^{3}$ \\
\hline Embryo & $75.7 \pm 6.3$ & $1.5 \pm 0.2$ & 0.0 \\
\hline Aleurone layer & $55.3 \pm 4.5$ & $1.2 \pm 0.3$ & 0.0 \\
\hline Endosperm & 0.0 & 0.0 & 0.0 \\
\hline
\end{tabular}

${ }^{1}$ nmol phenantridone $\mathrm{mg}^{-1}$ protein $\min ^{-1} ;{ }^{2} \mu \mathrm{mol} \mathrm{NADH} \mathrm{mg}{ }^{-1}$ hour ${ }^{-1} ;{ }^{3} \mu \mathrm{mol} \mathrm{NO} \mathrm{Ng}^{-1}$ hour ${ }^{-1}$

Low AO activity was detected in the embryo and aleurone layer of seeds until the age of 30 DPA. After this age the activity of the enzyme in the embryo increased steeply reaching a maximum at 40 DPA, the enzyme activity in the aleurone layer increased slightly. Thereafter, AO activity in the embryo fell significantly at full maturity of the seeds, while AO activity in the aleurone layer remained at a steady level.

Table 2 - Changes in AO, XDH and NR activities in seed embryo during seed development and maturation

\begin{tabular}{|c|c|c|c|c|c|c|c|}
\hline $\mathrm{DPA}$ & 10 & 20 & 30 & 40 & 50 & 60 & FM \\
\hline $\mathrm{AO}$ & $10.2 \pm 1.7$ & $11.8 \pm 2.1$ & $18.3 \pm 2.4$ & $67.4 \pm 8.7$ & $65.7 \pm 9.3$ & $66.4 \pm 7.4$ & $66.8 \pm 7.4$ \\
\hline $\mathrm{XDH}$ & $16 \pm 0.2$ & $1.6 \pm 0.2$ & $1.7 \pm 0.3$ & $1.8 \pm 0.2$ & $1.9 \pm 0.3$ & $1.9 \pm 0.2$ & $1.9 \pm 0.2$ \\
\hline $\mathrm{NR}$ & 0.0 & 0.0 & 0.0 & $0.3 \pm 0.02$ & $2.3 \pm 0.1$ & $4.7 \pm 0.5$ & $5.1 \pm 0.7$ \\
\hline
\end{tabular}

As can be seen from the Figure 2, the activity of AO is beginning to show up in the initial period of maturation (25DAP), and in all parts of the seed. 35-40 days in the endosperm, and particularly in the germinal part of the activity of the enzyme increases about 10 times compared with the early stages of ripening grains. In electrophoretic spectrum first appear cathodic components (AO1) involved in the biosynthesis of ABA (30 DAP). 
DAP

Parts of seeds

Total Relative Intensity (\%)

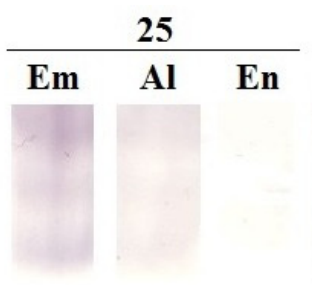

$48 \quad 16$

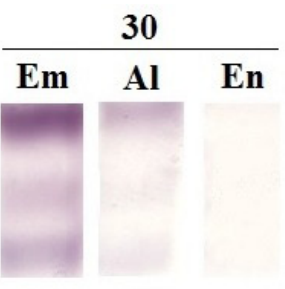

$\begin{array}{lll}108 & 45 & 0\end{array}$

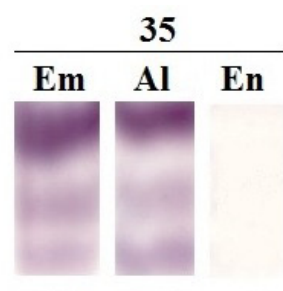

$\begin{array}{lll}122 & 119 & 0\end{array}$

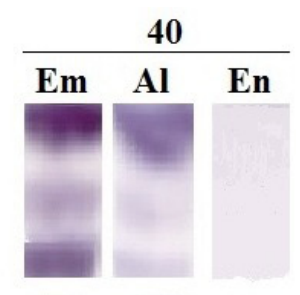

$\begin{array}{lll}134 & 115 & 0\end{array}$

Figure 2 - The activity of AO in different parts of developing and maturing seeds of the wheat cultivar Lutescens 70 (Em - embryo, Al - aleurone layer, En - endosperm)

In subsequent phase of ripening the increase in the activity of AO is due to a cathodic activation of the enzyme and the appearance in the spectrum of the anode forms (AO1 and AO3).

Approximately the 40th DAP fresh weight of the grain reaches a maximum and then begins the stage of dehydration or drying and, at this time gradually decreases the level of the AO. It should be noted that AO1 throughout the ripening period is a major component in the germ.
Studied the relationship between ABA content, dry weight and ability to germinate in seeds of examined wheat varieties resistant to preharvest sprouting Lutescens 70, and unstable - Novosibirskaya 67.

The data obtained are shown in Table 3, from which it follows that the level of ABA after reaching its maximum of 40-45 days began quickly to decline towards the end of ripening process (55 DAP). Tabular data show a marked increase in ability to germinate, starting from the phase of 40-45 DAP.

Table 3 - Dynamics of changes in ABA and the ability to germinate wheat seeds at different stages of maturation

\begin{tabular}{|c|c|c|c|c|c|c|}
\hline \multirow{2}{*}{ DAP } & \multicolumn{3}{|c|}{ Lutescens 70 } & \multicolumn{3}{c|}{ Novosibirskaya 67 } \\
\cline { 2 - 7 } & $\begin{array}{c}\text { ABA } \\
\text { ng/mg dried } \\
\text { weight }\end{array}$ & \% germination & $\begin{array}{c}\text { Dried weight, } \\
\%\end{array}$ & $\begin{array}{c}\text { ABA } \\
\text { ng/mg of dried } \\
\text { weight }\end{array}$ & \% germination & $\begin{array}{c}\text { Dried weight, } \\
\%\end{array}$ \\
\hline 20 & 0.70 & 0.0 & 35 & 0.45 & 0.0 & 37 \\
\hline 25 & 0.65 & $3-5$ & 50 & 0.45 & $6-8$ & 40 \\
\hline 30 & 0.75 & $7-10$ & 65 & 0.45 & $10-13$ & 56 \\
\hline 35 & 0.80 & $11-12$ & 75 & 0.55 & $13-16$ & 75 \\
\hline 40 & 0.95 & $10-11$ & 89 & 0.60 & $15-19$ & 86 \\
\hline 45 & 0.85 & $14-16$ & 92 & 0.60 & $22-30$ & 88 \\
\hline 50 & 0.60 & 20 & 95 & 0.40 & $32-45$ & 92 \\
\hline 55 & 0.60 & $22-25$ & 98 & 0.35 & $40-60$ & 95 \\
\hline $\begin{array}{c}\text { Complete } \\
\text { germination }\end{array}$ & 0.50 & 25 & $100^{*}$ & 0.30 & $55-65$ & 95 \\
\hline
\end{tabular}

* $100 \%$ - dry weight of 50 seeds

Such temporal coincidence, apparently, is not accidental, given the fact that $\mathrm{ABA}$ is an important endogenous regulator (inhibitor) of germination. May decrease endogenous $\mathrm{ABA}$ content in the grains at the final stage of ripening, with relatively high germination percentage can be explained by the need and ensuring to the start of the germination process of the seed.
There was no NR activity detectable in vivo and in vitro in the embryo, endosperm and aleurone layer of developing seeds. In contrast, when seeds were removed from the spikes that were at different stages of maturation and incubated in $50 \mathrm{mM} \mathrm{KNO}$ solution for 30 hours, NR activity was detected in embryos starting at 40 DAP (Table 2). 
The maximum level of NR activity was observed in the embryo portion of mature seeds.

NR activity in germinating seeds of PHS-tolerant and sensitive wheat cultivars. The following experiments were carried out to study the activities of NR in pre-harvest sprouting tolerant and sensitive wheat varieties during their germination. The seeds were separated into aleurone layer, endosperm and embryo. These parts of dry seeds showed no detectable NR activity. Seeds were incubated in distilled water for 24-30 h, then separated and into their parts to determine NR activity in each of the tissues. NR activity appeared in seed embryo only after incubating them in the presence of $\mathrm{KNO}_{3}$ and its levels varied among wheat varieties (Table 3 ).

Seeds were incubated in nitrate and at appropriate times embryos were removed from the seeds to estimate the enzyme activity. In the present set of experiments embryos were first excised and then incubated in nitrate. Study of NR induction in the excised embryos showed a significant level of the NR activity as early as $6 \mathrm{~h}$ of germination.

Table 4 - NR activity of attached and detached seed embryos of PHS-tolerant Lutescence 70 and PHS-sensitive Novosibirskaya 67 wheat varieties

\begin{tabular}{|c|c|c|c|c|c|c|c|}
\hline \multirow{2}{*}{$\begin{array}{c}\text { Wheat } \\
\text { varieties }\end{array}$} & \multirow{2}{*}{$\begin{array}{l}\text { Type of } \\
\text { embryo }\end{array}$} & \multicolumn{6}{|c|}{ Incubation time, $\mathrm{h}$} \\
\hline & & 0 & 6 & 12 & 18 & 24 & 30 \\
\hline \multirow{2}{*}{$\begin{array}{l}\text { PHS- } \\
\text { tolerant }\end{array}$} & Attached & 0.0 & 0.0 & 0.0 & 0.0 & 0.0 & 0.0 \\
\hline & Detached & 0.0 & $2.7 \pm 0.2$ & $4.3 \pm 0.7$ & $3.2 \pm 0.5$ & $2.5 \pm 0.4$ & $1.8 \pm 0.2$ \\
\hline \multirow{2}{*}{ PHS-sensitive } & Attached & 0.0 & $0.5 \pm 0.1$ & $1.2 \pm 0.1$ & $2.4 \pm 0.3$ & $1.9 \pm 0.3$ & $1.3 \pm 0.2$ \\
\hline & Detached & 0.0 & $3.2 \pm 0.4$ & $3.2 \pm 0.4$ & $4.8 \pm 0.6$ & $4.2 \pm 0.5$ & $3.6 \pm 0.6$ \\
\hline
\end{tabular}

After this, the enzyme activity increased and showed a peak at $12 \mathrm{~h}$ of incubation followed by a decrease in the level of the enzyme activity which came to the minimum activity after $30 \mathrm{~h}$ of incubation (Table 4). It is interesting to note that the embryos of ungerminated wheat seeds of both varieties could not synthesize NR in the presence of $60 \mathrm{mM} \mathrm{KNO}_{3}$ after $12 \mathrm{~h}$ induction. Since wheat seeds germinate in the absence of nitrate and without NR synthesis, seed germination is not depended on nitrate assimilation in the embryo.

Since in PHS-tolerant seeds the presence of the endosperm delayed NR induction, attempts were made to study effects of cross-combinations of PHStolerant and PHS-sensitive endosperm extracts on the induction of NR in their detached embryos.

Results of these experiments are shown in Table 5 .

Table 5 - Effects of cross-combinations of fresh endosperm extracts of PHS-tolerant and sensitive on NR-induction in their detached embryos in after $12 \mathrm{~h}$ induction presence of $60 \mathrm{mM}$

\begin{tabular}{|l|c|}
\hline \multicolumn{1}{|c|}{ Combinations of endosperm extracts and detached embryos in $\mathrm{KNO}_{3}$} & $\begin{array}{c}\text { NR activity in detached } \\
\text { embryos }\end{array}$ \\
\hline Detached embryo of PHS-tolerant seed in & $4.6 \pm 0.9$ \\
\hline Endosperm of PHS-tolerant seed + its detached embryo & $0.5 \pm 0.1$ \\
\hline Detached embryo of PHS-sensitive seed & $5.5 \pm 0.7$ \\
\hline Endosperm of PHS-sensitive seed + its detached embryo & $2.9 \pm 0.6$ \\
\hline Endosperm of PHS-tolerant seed + detached embryo of PHS-sensitive seed & $1.9 \pm 0.3$ \\
\hline Endosperm of PHS-sensitive seed + detached embryo of PHS- tolerant seed & $0.8 \pm 0.1$ \\
\hline
\end{tabular}


The endosperm of PHS-tolerant wheat seeds contains a factor inhibiting NR induction by nitrate in embryo, while this factor is not present in PHSsensitive cultivars.

This inhibitory factor present in the endosperm gets gradually destroyed with time (not shown).

Seed sensitivity of different wheat varieties to PHS depends on the levels of ABA in their endosperm and embryo, i.e. PHS-tolerant wheat seeds contain higher concentrations of ABA [21]. On the basis of these facts we proposed that ABA regulates the activity or synthesis of NR in wheat embryo. Detached embryos of PHS-tolerant seeds were incubated for $12 \mathrm{~h}$ in the presence of different ABA concentrations plus $60 \mathrm{mM} \mathrm{KNO}_{3}$.

Increasing concentrations of $\mathrm{ABA}$ increase the inhibition of NR activity in detached wheat embryo (Table 5).

Table 6 - Time course decrease of NR activity in PHS-tolerant embryo by increasing ABA concentrations (NR activity in nmoles of nitrite/embryo/h)

\begin{tabular}{|c|c|c|c|c|}
\hline ABA concentrations & Control & $10 \mathrm{nM}$ & $100 \mathrm{nM}$ & $1.0 \mu \mathrm{M}$ \\
\hline NR activity & $4.8 \pm 0.8$ & $4.3 \pm 0.8$ & $3.5 \pm 0.3$ & $1.3 \pm 0.2$ \\
\hline
\end{tabular}

Since ABA content in both seed types is nearly the same the endosperm of PHS-sensitive seeds may contain higher concentrations of ABA inactivating enzymes than the PHS-tolerant seeds, and this is one of reasons of PHS-sensitivity of wheat seeds. These observations are significant in view of the fact that $\mathrm{NR}$ is induced in wheat seed embryo linked to the seed tolerance to PHS. The inhibition of NR in detached embryos following 4-6 h of imbibition by endosperm extract may serve as an early marker of the tolerance of wheat seeds to PHS.

In the embryos present in the intact seed there was no NR activity until $24 \mathrm{~h}$ of incubation. It appears that the presence of endosperm in the intact seed inhibited the induction of NR in the embryos during the (first $24 \mathrm{~h}$ ) germination of the PHS-tolerant wheat seeds but not in the embryos of the PHSsensitive wheat seeds. Thus, only the endosperm of PHS-tolerant seed appeared to inhibit NR induction.

Other enzymes of nitrate assimilation pathway, such as nitrite reductase, glutamine synthase and glutamate dehydrogenase were also found to be present in in situ embryos during the initial stages of germination in wheat (not shown). Thus, it appears that among the key enzymes of nitrate assimilation only NR shows a long lag period in its development that seems to be exerted by the endosperm of PHS-sensitive seeds for NR.

The level of the inhibitory endosperm factor of PHS-sensitive wheat seeds appears to be lower in PHS-tolerant seeds. The inhibitory factor gradually disappears with seed aging, similarly to the fate of the dormancy controlling ABA, the level of which determines the PHS sensitivity of the seeds, suggesting that ABA may regulate the synthesis of NR in wheat embryos and perhaps in other plant tissues [22]. These observations are significant in view of the fact that NR induction in wheat seed embryos correlates with their level of PHS tolerance. Thus, the 4-6 $\mathrm{h}$ inhibition by the endosperm extract of NR in detached embryos may serve as a marker of the PHS tolerance of wheat seeds, and may be used as an early parameter for fast genetic screening.

\section{References}

1. Fang J. and Chu Ch. Abscisic acid and the pre-harvest sprouting in cereals. Plant Signal Behav. 2008. 3(12): 1046-1048.

2. Derera N.F. Preharvest Field Sprouting in Cereals. CRC Press Inc., 1989.

3. Curvers K., Seifi H., Mouille G., De Rycke R., Asselbergh B., Van Hecke A., et al. 2010. Abscisic acid deficiency causes changes in cuticle permeability and pectin composition that influence tomato resistance to Botrytis cinerea. Plant Physiol. 154 847-860 10.1104/pp.110.158972

4. Adie BAT, Perez-Perez J, Perez-Perez MM, Godoy M, Sanchez-Serrano JJ, Schmelz EA, Solano R. 2007. ABA is an essential signal for plant resistance to pathogens affecting JA biosynthesis and the activation of defenses in Arabidopsis. Plant Cell 19: 1665-1681

5. Walker-Simmons M. ABA levels and sensitivity in developing wheat embryos of sprouting resistant and susceptible cultivars . Plant Physiol. 1987. Vol. 84. pp. 41-46.

6. Steinbach H.S., Benech-Arnold R.L.,Sanches R.A. Hormonal regulation of dormancy in developing sorgum seeds.Plant Physiol. 1999. 113:149-154; 
7. Tanner P.D. A relationship between premature sprouting on the cob and molybdenum and nitrogen status of grain maize. Plant and Soil, 1978. 49:427432

8. Cairns A.L.P., Modi A.T.,Cowan A.K.Kritzinger J.H. The effects of molybdenum on seed dormancy In: R.H.Ellis,M.Black, Murdoch A.J. and Hong T.D. eds. Basic and Applied Aspects of seed biology. Kluwer Academic Publishers, 1997. pp.173-181.

9. Leydecker, M.T., Moureaux, T., Kraepiel, Y., Schnorr, K., Caboche, M. Molybdenum cofactor mutants, specifically impaired in xanthine dehydrogenase activity and abscisic acid biosynthesis, simultaneously overexpress nitrate reductase. Plant Physiology, 1995, 107, 1427-1431.

10. Parry, A.D., Neill, S.J., Horgan, R. Xantoxin levels and metabolism in the wild-type and wilty mutants of tomato. Planta, 1988. 173, 397-404.

11. Schwarz, G., Mendel, R.R., Ribbe, M.W. Molybdenum cofactors, enzymes and pathways. Nature, 2009. 460, 839-847.

12. Kruse T., Gehl C., Geisler M., Lehrke M., Ringel P., Hallier S., Hansch R., Mendel R.R. Identification and biochemical characterization of molybdenum cofactor-binding proteins from Arabidopsis thaliana. Journal of Biological Chemistry, 2010. 285, 6623-6635

13. Sekimoto, H., Seo, M., Dohmae, N., Takio, K., Kamiya, Y., Koshiba, T. Cloning and molecular characterization of plant aldehyde oxidase. J. Biol. Chem., 1997. 272, 15280-15285.

14. Ori, N., Eshed, Y., Pinto, P., Paran, I., Zamir, D., Fluhr, R. TAO1, a representative of molybdenum cofactor containing hydroxylases from tomato. J. Biol. Chem., 1997. 272, 1019-1025.

15. Terao M., Kurosaki M., Saltini G., Demontis S., Marini M., Salmona M., Garattini E. Cloning of the cDNA for two aldehyde oxidase and xanthine oxidoreductase. J.Biol.Chem. 2000. 275 (39): 3069030700

16. Fang, J., Chai, C., Qian, Q., Li, C., Tang, J., Sun, L., Huang, Z., Guo, X., Sun, C., Liu, M. Mutations of genes in synthesis of the carotenoid precursors of ABA lead to pre-harvest sprouting and photooxidation in rice. Plant Journal, 2008. 54, 177-189.

17. Gerjets T.; Scholefield D.; Foulkes M.J.; Lenton J.R.; Holdsworth M.J. An analysis of dormancy, ABA responsiveness, after-ripening and pre-harvest sprouting in hexaploid wheat (Triticum aestivum L.) caryopses. Journal of Experimental Botany, 2010. 61, 597-607.

18. Savidov, N.A., Alikulov, Z., Lips, H. Identification of an endogenous NADPH-regenerating system coupled to nitrate reduction in vitro and fungal crude extracts. Plant Science, 1998. 133(1), 33-45.

19. Barabas N.K., Omarov R.T., Erdei L., Lips S.H. Distribution of the Mo-enzymes aldehyde oxidase, xanthine dehydrogenase and nitrate reductase in maize (Zea mays L.) nodal roots as affected by nitrogen and salinity. Plant science, 2000. 155, 49-58

20. Alikulov Z., Schieman. Presence of active molybdenum cofactor in dry seeds of wheat and barley. Plant Sci. 1985. 40. 161-165.

21. Kawakami N, Miyake Y., Kzuhiko N. ABA insensitivity and low ABA levels during seed development of non-dormant wheat mutants. J. Exp. Botany. 1997. 48(312): 1415-1421.

22. Finch-Savage W.E., Cadman C.S.C., Toorop P.E., Lynn J.R., Hilhorst H.W.M. Seed dormancy release in Arabidopsis by dry after-ripening, low temperature, nitrate and light shows common quantitative patterns of gene expression directed by environmentally specific sensing Plant Journal, 2007. 51, 60-78. 